\title{
Graphene-Quantum Dot Hybrid Optoelectronics at Visible Wavelengths
}

\author{
Omer Salihoglu, ${ }^{\dagger}$ Nurbek Kakenov, ${ }^{\dagger}$ Osman Balci, ${ }^{\dagger}$ Sinan Balci, ${ }^{\ddagger}{ }^{\ddagger}$ and Coskun Kocabas ${ }^{*},,^{\dagger} \S_{\odot}$ \\ ${ }^{\dagger}$ Department of Physics, Bilkent University, 06800 Ankara, Turkey \\ ${ }^{\ddagger}$ Department of Photonics, Izmir Institute of Technology, 35430 Izmir, Turkey \\ ${ }^{\S}$ School of Materials, National Graphene Institute, University of Manchester, M13 9PL Manchester, United Kingdom
}

\section{Supporting Information}

\begin{abstract}
With exceptional electronic and gate-tunable optical properties, graphene provides new possibilities for active nanophotonic devices. Requirements of very large carrier density modulation, however, limit the operation of graphene based optical devices in the visible spectrum. Here, we report a unique approach that avoids these limitations and implements graphene into optoelectronic devices working in the visible spectrum. The approach relies on controlling nonradiative energy transfer between colloidal quantum-dots and graphene through gate-voltage induced tuning of the charge density of graphene. We demonstrate a new class of large area optoelectronic devices including fluorescent display and voltage-controlled color-variable devices working in the visible spectrum. We anticipate that the presented technique could provide new practical routes for active control of light-matter interaction at the nanometer scale, which could find new implications ranging from display technologies to quantum optics.
\end{abstract}
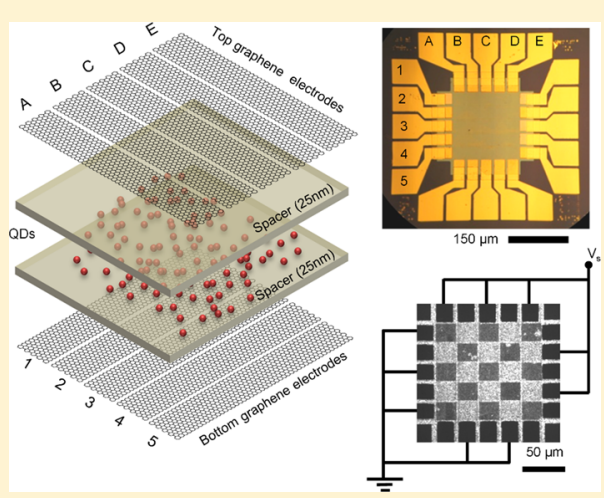

KEYWORDS: graphene, quantum dots (QDs), optoelectronics, nanophotonics, graphene field effect transistors (GFETs), heterostructures, and fluorescent displays

$\mathrm{C}$ ontrolling light-matter interactions at the nanometer length scale is a central goal of nanophotonics. ${ }^{1}$ Plasmonic nanostructures, ${ }^{2,3}$ photonic crystals, ${ }^{4}$ and quantumwells $s^{5}$ have been used to manipulate these interactions. Small electro-optic effects ${ }^{6-8}$ and the lack of electric-field effect in metals have been the challenges hindering active control of nanophotonic devices. Very recently, graphene has provided a new flexible platform for gate-controlled optical devices such as optical modulators, ${ }^{9-11}$ tunable photonic microcavities, ${ }^{12,13}$ and plasmonic devices. ${ }^{14-17}$ Indeed, these applications are mainly based on Pauli blocking principle, ${ }^{18-21}$ that is, the optical absorption of graphene can be blocked when the gate-induced shift of Fermi energy is larger than the half of the excitation energy. However, the working wavelength of these devices is limited to infrared wavelengths. ${ }^{16}$ In order to suppress the interband transitions in the visible spectrum, the Fermi level needs to be modulated larger than $1 \mathrm{eV}$, which requires extremely large gate-induced carrier densities or extreme doping of graphene $\left(>5 \times 10^{13} \mathrm{~cm}^{-2}\right) \cdot{ }^{15,19}$ In fact, these electrostatic doping levels are not practical with conventional gate dielectric materials due to the dielectric breakdown. On the one hand, electrolyte-based gating schemes can decrease the working wavelengths down to near-infrared region, ${ }^{12,15,19,22}$ but on the other hand, requirements of liquid medium and limited switching time of electrolytes significantly hinder the realization of the full potential held by graphene for optoelectronic applications in the visible spectrum.
To overcome these limitations, we used gate-controlled fluorescence quenching of colloidal quantum-dots (QDs) embedded in the dielectric layer of graphene transistors. Since the rate of nonradiative energy transfer between QDs and graphene depends on the concentration of free carriers in graphene, $^{22-25}$ the fluorescence of QDs can be controlled through gate-induced carriers in graphene. Our method is suitable for visible spectrum defined by the emission of the QDs. Colloidal quantum-dots have enabled applications ranging from biological imaging to display technologies ${ }^{26}$ owing to their high quantum yields, tunable and narrow spectral emission, and photostability. ${ }^{27}$ Various excitation mechanisms of QDs such as optical excitations, charge injection, and energy transfer have been developed for lightemitting applications. ${ }^{27}$ The lack of efficient electrical control of the emission of QDs has been the challenge delaying the full potential of quantum-dots for active optoelectronic devices. Recently, energy transfer between two-dimensional sheets of graphene and zero dimensional quantum dots have been theoretically and experimentally studied. ${ }^{22,28-32}$ Integrating QDs with graphene transistors yields a new class of hybrid devices that enable gate-controlled fluorescence which could be suitable for basic research and applied technologies. Unlike the

Received: February 5, 2018

Published: April 20, 2018 

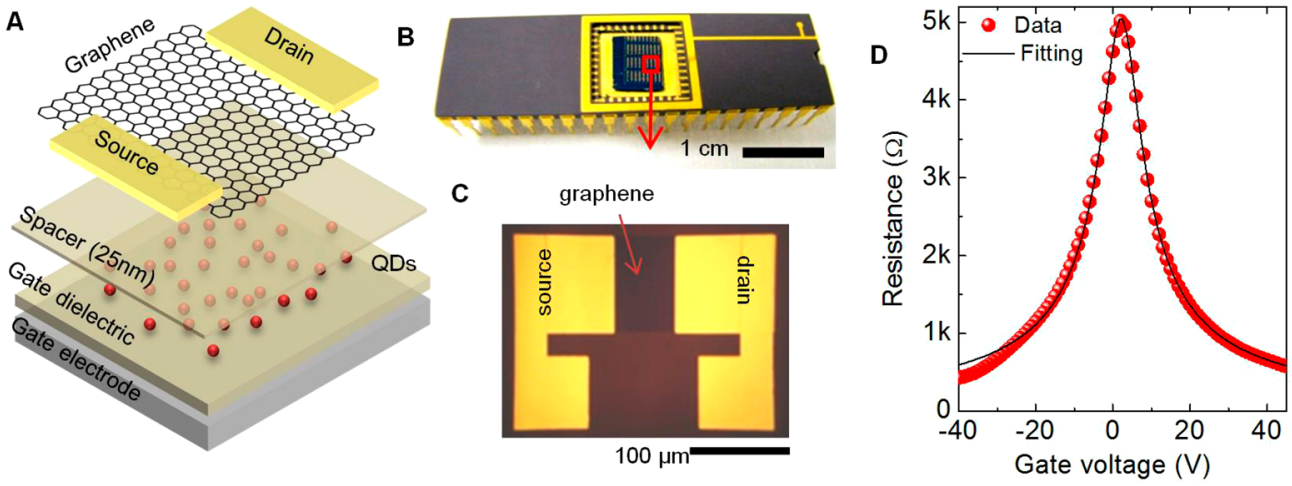

Figure 1. Graphene field-effect transistors integrated with QDs embedded in the dielectric layer. (a) Schematic shows the exploded view of the backgated graphene field effect transistor. The QDs are sandwiched between two dielectric layers $(65$ and $25 \mathrm{~nm})$ and graphene is transfer-printed on the top dielectric layer. (b) Photograph of the fabricated transistors wire bonded on a DIP chip holder. (c) Optical microscope image of a graphene-FET. (d) Gate-dependent electrical resistance of graphene. The black line represents the fitting curve. The resistance reaches its maximum value at the charge neutrality (Dirac) point $V_{\mathrm{CNP}}=2 \mathrm{~V}$. The on-off ratio of the transistor is around 10 .
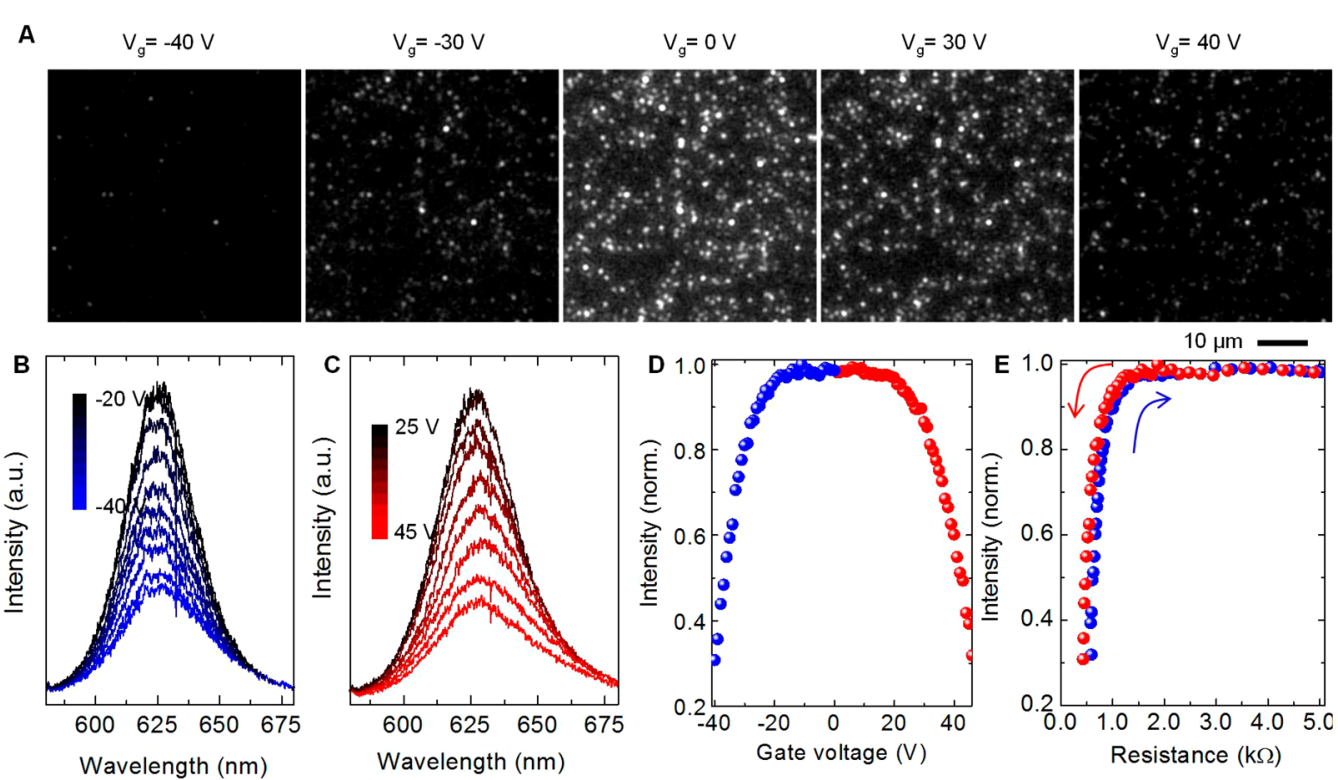

Figure 2. Gate-controlled fluorescence quenching of QDs. (a) Fluorescence microscope images of the QDs in the channel area of graphene transistors for various gate voltages ranging from -40 to $40 \mathrm{~V}$. (b, c) The quenching of QD fluorescence for hole (blue) and electron (red) transport regimes as the gate voltage varies from -40 to $-20 \mathrm{~V}$ and 25 to $45 \mathrm{~V}$, respectively. (d) Gate-dependent fluorescence intensity of QDs in the channel area for negative and positive voltages. The gate voltage was swept from $-40 \mathrm{~V}$ to $+45 \mathrm{~V}$. Blue and red color represent hole and electron transport regimes, respectively. (e) Correlation between the measured fluorescence intensity and the resistance of the graphene. The arrows indicate the direction of the voltage sweep.

previous studies, we here demonstrate solid state scheme to control fluorescence of QDs and new optoelectronic devices working in the visible part of the electromagnetic spectrum. Our work has four novel aspects: (i) we demonstrate a new fluorescence quenching scheme using graphene field effect transistors, (ii) we demonstrate electrical control of fluorescence intensity and lifetime of QDs, (iii) we show that chemical doping of graphene can be used to control florescence of QDs, and (iv) we implement gate tunable quenching of QDs for new type of hybrid displays and color-variable devices working in the visible spectrum.

We integrated colloidal QDs with back-gated graphene fieldeffect transistors. We used $\mathrm{CdSe} / \mathrm{ZnS}$ (Qdot 625, Catalog Number A10197) with emission maxima at $\sim 625 \mathrm{~nm}$, purchased from Life Technologies Corporation. We drop casted the diluted $4 \mathrm{nM}$ of quantum-dot solution on the device surface. Figure la shows the device layout. The gate dielectric consists of two layers of $\mathrm{Si}_{3} \mathrm{~N}_{4}(65 \mathrm{~nm}$ bottom layer and $25 \mathrm{~nm}$ top layer) grown by chemical vapor deposition. Graphene was synthesized on ultrasmooth copper foils (Mitsui Mining and Smelting Co., Ltd., B1-SBS) by chemical vapor deposition at $1035^{\circ} \mathrm{C}$, as reported in our previous publications. ${ }^{33}$ After the transfer-printing process of graphene, we fabricated source and drain electrodes with standard UV-photolithography and performed two layers of metallization process (SI, Figures 16). In order to perform simultaneous electrical and optical measurements, the devices were wire-bonded on conventional ceramic chip holders. Figure 1b,c shows the device mounted on a chip holder and a zoomed image of an individual graphene transistor. The transistor has a channel width of $100 \mu \mathrm{m}$ and a channel length of $64 \mu \mathrm{m}$. The critical design aspects of the devices include optimum dielectric thickness with very low 

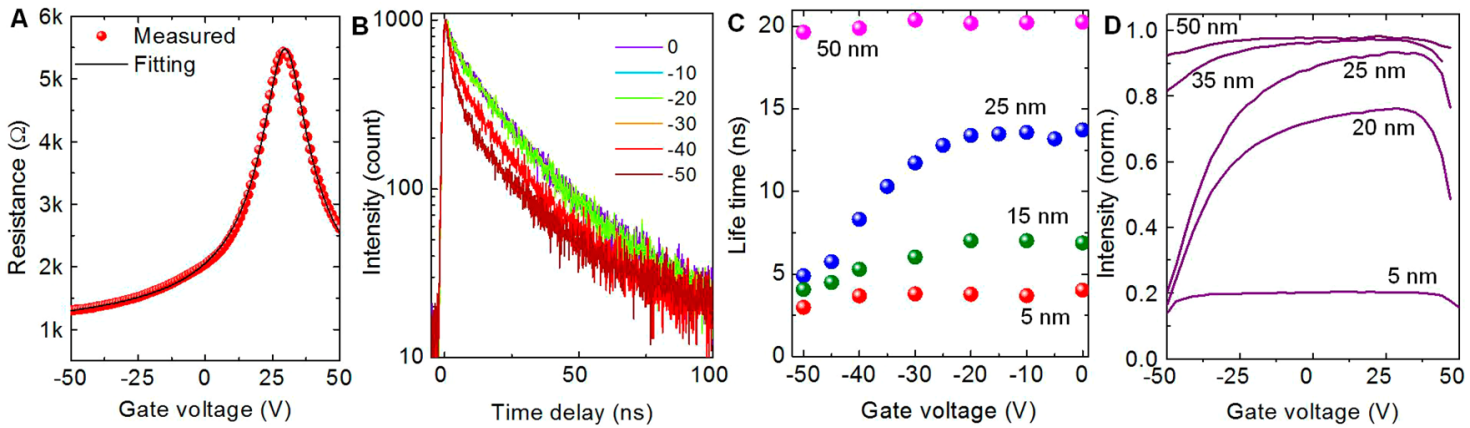

Figure 3. Gate-controlled fluorescence lifetime of QDs. (a) Gate voltage-dependent electrical resistance of the large area graphene transistor. (b) The lifetime trajectories of QDs for various gate voltages from 0 to $-50 \mathrm{~V}$. The dependence of extracted lifetime (c) and fluorescence intensity (d) of $\mathrm{QDs}$ on the gate voltages between -50 to $50 \mathrm{~V}$ for various spacer layer thicknesses.

gate-leakage current (less than $1 \mathrm{nA}$ ), and deposition of the dielectric on QDs without significant detrimental effects on the fluorescence. Figure 1d shows the gate voltage dependent electrical resistance of graphene. The resistance of graphene reaches its maximum value (around $5 \mathrm{k} \Omega$ ) at the charge neutrality (Dirac) point $V_{\mathrm{CNP}}=2 \mathrm{~V}$ and on-off ratio around 10. The resistance of graphene can be expressed as $R_{\mathrm{T}}=2 R_{\mathrm{c}}+\frac{L}{W \mu \sqrt{n_{0}^{2}+n_{\mathrm{g}}^{2}}}$, where $R_{\mathrm{T}}$ is the total resistance, $R_{\mathrm{c}}$ is the contact resistance, $L$ and $W$ are the width and the length of the channel, $\mu$ is the carrier mobility, $n_{0}$ is the minimum carrier density, and $n_{\mathrm{g}}$ is the gate induced carrier density. The $n_{\mathrm{g}}$ is tuned by the gate voltage $V_{\mathrm{g}}$ as $n_{\mathrm{g}}=\frac{C_{\mathrm{g}}\left(V_{\mathrm{g}}-V_{\mathrm{CNP}}\right)}{e}$, where $C_{\mathrm{g}}$ is the gate capacitance and $e$ is the elementary charge. The fit of the resistance (black solid line in Figure 1d) yields the field effect mobility of $600 \mathrm{~cm}^{2} /(\mathrm{V} \mathrm{s})$, and $n_{0}$ of $1.5 \times 10^{12} \mathrm{~cm}^{-2}$.

We swept the gate voltage from $-40 \mathrm{~V}$ to $+45 \mathrm{~V}$ and simultaneously recorded the drain current and the fluorescence images of the QDs using a Nikon inverted fluorescence microscope equipped with Hamamatsu EMCCD camera, (see SI, Figure 1 for details). Electrical measurements were performed using Keithley Model 2400 and 2401 Source Meter Instrument. Gray scale fluorescence images of QDs at five representative gate voltages are given in Figure $2 \mathrm{a}$. We observed strong fluorescence quenching at both positive and negative gate voltages owing to the ambipolar charge transport properties of graphene (SI, Movie 1).The fluorescence spectra in Figure 2b,c show the gate-controlled quenching for hole and electron transport regimes (SI, Figure 7). We did not observe a significant spectral shift $(<2 \mathrm{~nm})$ or broadening in the emission spectrum. The voltage dependence of the fluorescence intensity of the QDs (averaged over the channel area) is given in Figure $2 \mathrm{~d}$. The blue and red dots represent the hole and electron transport regimes, at negative and positive gate voltages, respectively. Besides, we observed a plateau in the fluorescence intensity for the gate voltages ranging from $-20 \mathrm{~V}$ to $+20 \mathrm{~V}$. The correlation of the fluorescence intensity and resistance of the graphene layer is shown in Figure 2e. Indeed, the fluorescence is significantly quenched by graphene when the resistance is less than $1 \mathrm{k} \Omega$ (corresponding to charge density of $5 \times 10^{12} \mathrm{~cm}^{-2}$ ). To gain more insight into the correlation between resistivity and fluorescence quenching, we performed similar experiments with a p-type graphene transistor with a charge neutrality point at large positive voltages (SI, Figure 5 and SI, Movie 2). Unlike the ambipolar transistors, we observed fluorescence quenching only for the negative voltages. These observations suggest that the fluorescence quenching is proportional to the carrier density in graphene.

In order to clarify the dominant energy transfer mechanism involved in the gate-controlled quenching, we measured gate voltage-dependence of fluorescence lifetime of the QDs. To perform these experiments, we fabricated large area graphene transistors with similar layout shown in Figure 1a but the transistors have a large channel area $\left(\sim 1 \mathrm{~cm}^{2}\right.$, SI, Figure 6). The transfer curve of the device is shown in Figure 3a. We monitored the temporal evolution of fluorescence using a timecorrelated single photon counting system as we tuned the gate voltage. Time-correlated single photon counting experiments were performed using Horiba, NanoLog series of spectrafluorometers equipped with Fluoro3PS-Photomultiplier Power Source. NanoLED laser diode with excitation wavelength of $390 \mathrm{~nm}$ at repetition rates of $1 \mathrm{MHz}$ is used to excite QDs. The lifetime trajectories of QDs were recorded for $400 \mathrm{~ns}$ time durations. In fact, Figure $3 \mathrm{~b}$ shows the fluorescence dynamics of QDs embedded in the dielectric of graphene transistor for various gate voltages. After fitting the lifetime trajectories, we obtained the lifetime of QDs as a function of gate voltage, Figure 3c. For the transistor with $25 \mathrm{~nm}$ spacer layer, we observed a strong decrease in the lifetime from 15 to $5 \mathrm{~ns}$ for the negative gate voltages owing to the large carrier injection. The variation of fluorescence intensity of the devices with different dielectric thicknesses is plotted in Figure 3d. Since the transistor shows asymmetric ambipolar transport behavior with large positive charge neutral point, the decrease in the lifetime in the electron injection regime is relatively small owing to the less gate-induced electron density.

The hallmark of Förster type energy transfer is the scaling of the fluorescence with the distance from the surface of the acceptor. The measured Förster critical distance $\left(d_{0} \approx 19 \mathrm{~nm}\right)$ defines the length scale in our system. We performed similar type of measurements with various spacer thicknesses (see Figure $3 c, d)$. When the QDs are very close to the graphene surface $\left(<d_{0}\right)$, the fluorescence is significantly quenched with a weak gate dependence. Whereas, for a distance of $50 \mathrm{~nm}\left(>d_{0}\right)$, there is a very weak quenching and weak gate dependence. Maximum gate-induced modulation of fluorescence intensity and lifetime is obtained at a spacer distance around $25 \mathrm{~nm}$ $\left(\sim d_{0}\right)$.

One remaining question is whether the observed fluorescence quenching of QDs is associated with the electric field on the QDs. To clarify this ambiguity, we performed a controlled experiment using chemical doping, which provides similar level of charge densities but without electric field on QDs covered 

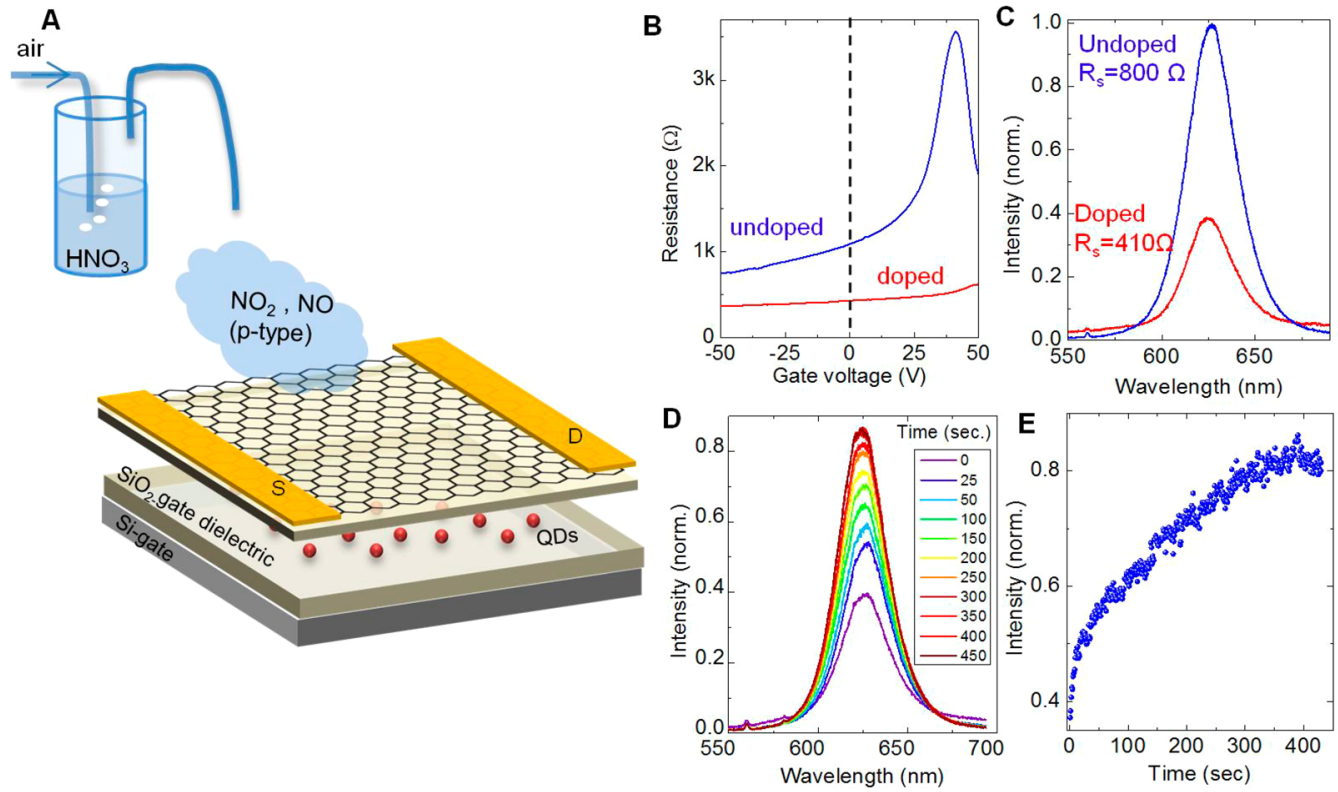

Figure 4. Controlling fluorescence quenching with chemical doping of graphene. (a) Schematic view of the experimental setup used for chemical doping of graphene transistors integrated with QDs. (b) Transfer curves of the graphene transistor at undoped and chemically doped conditions. (c) Recorded emission spectra of the QDs for undoped $\left(R_{\mathrm{s}}=800 \Omega\right)$ and doped $\left(R_{\mathrm{s}}=410 \Omega\right)$ conditions. (d) Recorded emission spectra of the QDs as the adsorbed chemicals desorb from the graphene surface. (e) The recovery of the fluorescence of QDs during desorption of chemical dopants.

with dielectric spacer layer thickness of $25 \mathrm{~nm}$. We used the same back-gated graphene field effect transistor configuration and then controlled the carrier density on graphene with chemical doping. Figure 4a shows the schematic representation of the experimental system used for controlling the doping on graphene with nitric acid vapor, which introduces strong p-type doping. ${ }^{34}$ Figure $4 \mathrm{~b}$ shows the transfer curves of the transistor before and after the chemical doping. After exposing the graphene transistor with nitric acid vapor, graphene becomes highly p-doped (charge neutrality point shifts to large positive voltages) due to the adsorbed electron accepting radicals (i.e., $\mathrm{NO}_{2}$ or $\mathrm{NO}_{3}$ ). However, the doping process contains volatile chemicals desorbing from the device surface, which allow us to recover the initial undoped state. After exposing the device to the acid vapor, we observed a significant reduction in the fluorescence intensity of QDs (Figure 4c). To quantify the doping level, we measured the resistance of the device at $V_{\mathrm{g}}=0$ $\mathrm{V}$. While the sheet resistance of graphene reduces from 800 to $410 \Omega,^{34}$ fluorescence intensity decays concurrently one-third of its initial value. The chemical doping on graphene is unstable and electrical properties of graphene vary over time. The fluorescence intensity recovers back to its initial value as the adsorbed dopants leave the graphene surface. Figure 4d,e shows the recovery of the fluorescence spectra and intensity of QDs.

To further understand the mechanism of the fluorescence quenching, we performed control experiments in the absence of graphene by replacing graphene with indium thin oxide (ITO). ITO yields a metallic conducting channel with negligible gate tunability. We measured the florescence intensity of QDs integrated with the gate-dielectric where the ITO is the channel material. We do not observe any modulation in the fluorescence intensity (see SI, Figure 8). Accordingly, the strong dependence of fluorescence quenching on the Dirac point, the critical dependence on graphene-QD distance together with the chemical doping, and the control experiments with ITO electrodes further support that the fluorescence of QDs can be tuned by changing the charge density on nearby graphene. It should be emphasized that the interband transition generates an electron-hole pair in the conduction and valence bands. Large shift in Fermi energy $(>1 \mathrm{eV})$ can block the interband transition and reduces the fluorescence quenching. ${ }^{28,32}$ However, here in this study, the Fermi energy is less than $0.4 \mathrm{eV}$ (SI, Figure 7), therefore the decay rate associated with the interband transition cannot be tuned by the gate voltage.

To show the promises of the method, we demonstrated a new class of optoelectronic devices working in the visible spectrum. Sandwiching QDs between two graphene electrodes in array geometry provides a new design for a display device. Figure 5 outlines the design and characterization of the fabricated display device. The exploded view of the $5 \times 5$ passive matrix display is given in Figure 5a. The device has a simple design with 5 bottom graphene electrodes and 5 top electrodes patterned into a ribbon shape with a width of $30 \mu \mathrm{m}$ and a length of $200 \mu \mathrm{m}$. The graphene electrodes were patterned by UV-photolithography and $\mathrm{O}_{2}$ plasma etching (SI, Figure 8). A $25 \mathrm{~nm}$ thick dielectric layer was used to control the QD-graphene distance. Optical micrograph of the fabricated device is shown in Figure 5b. The gold contact pads in both sides of the graphene ribbons are used to apply voltage bias to address the pixels. After wire bonding the display on a chip holder, we recorded the fluorescence image of QDs while applying a bias voltage to the electrodes. We first generated a static chessboard pattern by applying $20 \mathrm{~V}$ supply voltage, and $0 \mathrm{~V}$ to every other electrodes. The recorded fluorescence image of the chessboard pattern and wiring diagram are given in Figure 5c. The fluorescence of QDs is significantly quenched, when the voltage difference is larger than $12 \mathrm{~V}$ between the top and bottom graphene electrodes. The QDs at the intersections of electrodes with the same voltage bias yield a bright pixel owing to the high level of fluorescence. The fluorescence contrast changes as the supply voltage varies from $-20 \mathrm{~V}$ to $+20 \mathrm{~V}$ (SI, Movie 3). Figure 5d shows the fluorescence images of the display device at various supply voltages. The plot of the 

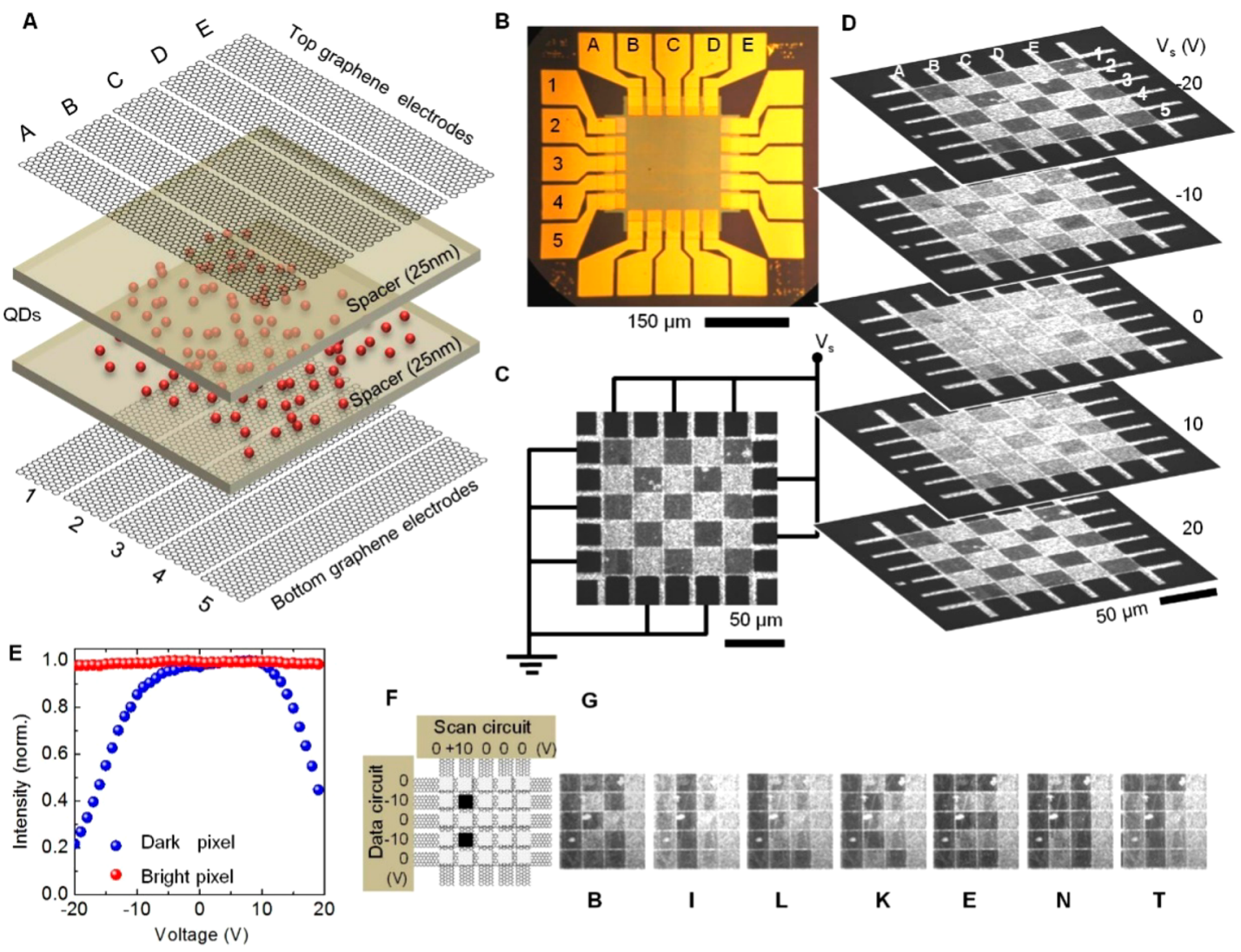

G
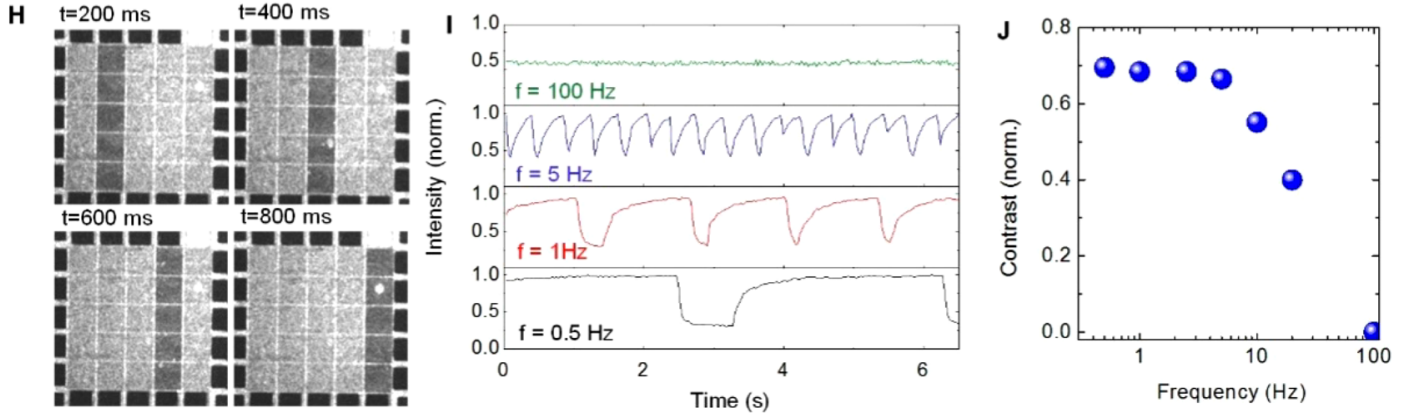

Figure 5. Graphene-based passive matrix fluorescent display. (a) Schematic shows the exploded view of the $5 \times 5$ passive matrix display based on graphene-enabled gate-controlled fluorescence. The letters and numbers label the top and bottom graphene electrodes, respectively. (b) Optical microscope image of the fabricated display device. The size of the pixels is $30 \mu \mathrm{m} \times 30 \mu \mathrm{m}$. (c) Fluorescence image (at $625 \mathrm{~nm}$ ) of the display biased to generate a chessboard pattern. (d) Fluorescence images of the display as the drive voltage, $V_{\mathrm{s}}$, varies from $-20 \mathrm{~V}$ to $+20 \mathrm{~V}$. (e) Normalized fluorescence intensity of a dark (red dots) and bright (blue dots) pixels as drive voltage $V_{\mathrm{s}}$ varies from $-20 \mathrm{~V}$ to $+20 \mathrm{~V}$. The asymmetry in the intensity is due to the shift in the charge neutrality point. (f) Schematic drawing of the electronic circuit used to generate dynamic fluorescence pattern on the display. To select a pixel (to quench the fluorescence of QDs), the circuit applies $-10 \mathrm{~V}$ to the bottom graphene electrode and $+10 \mathrm{~V}$ to the top graphene electrode while the rest of the electrodes are grounded. (g) Dynamic fluorescence pattern of 7 characters of the text "BILKENT". (h) Snapshots of the display with $200 \mathrm{~ms}$ time intervals as the scan circuits move the column from left to right while the data lines are biased to $-10 \mathrm{~V}$. (i) Time trace of a pixel with various switching time between $10 \mathrm{~ms}$ to $2 \mathrm{~s}$. (j) Generated fluorescence contrast of a pixel as a function of the switching frequency.

intensity of a bright and a dark pixel as a function of $V_{s}$ is shown in Figure 5e. The variation of the intensity profile resembles the results obtained in Figure 2. We observed that the charge neutrality point varies among the pixels resulting in some degree of inhomogeneity of the contrast. To generate dynamic images on the passive matrix display, we built a scan and data circuits. To select a pixel (to quench the fluorescence of QDs), we apply $+10 \mathrm{~V}$ to top electrode and $-10 \mathrm{~V}$ to the bottom electrode. Voltage difference of less than $12 \mathrm{~V}$ does not generate significant fluorescence quenching which yields a bright pixel on the display. We generated dynamic fluorescence patterns of seven characters of the text "BILKENT" (the name of our university). To generate the fluorescence image of a letter (Figure 5f,g), the circuit scans the columns with a scan rate of $100 \mathrm{~ms}$ and the data line refreshes the rows for every $100 \mathrm{~ms}$. The yield associated with transfer-printing process of graphene is quite high; however, there are some local defects that generate bright dots on the display. In addition, we observed some degree of hysteresis causing grayed appearance of the previous characters, which is most likely due to the charge injection into the dielectric. We tested the time response of the display device by changing the switching time of the 
drive circuit. Figure $5 \mathrm{~h}$ shows snapshot fluorescence images with $200 \mathrm{~ms}$ time interval. The time trace of a pixel for various switching times is plotted in Figure 5i. As the switching time decreases, the contrast between the bright and dark pixels decays. The hysteresis caused by the charge injection into the dielectric layer is likely due to the limiting mechanism of frequency response of the device. Figure $5 j$ shows the fluorescence contrast as a function of switching frequency (SI, Movie 4 and SI, Movie 5).

Furthermore, monolithic integration of QDs with different emission wavelength in a multilayer graphene device enables a new class of voltage-controlled color-variable devices. Figure 6a
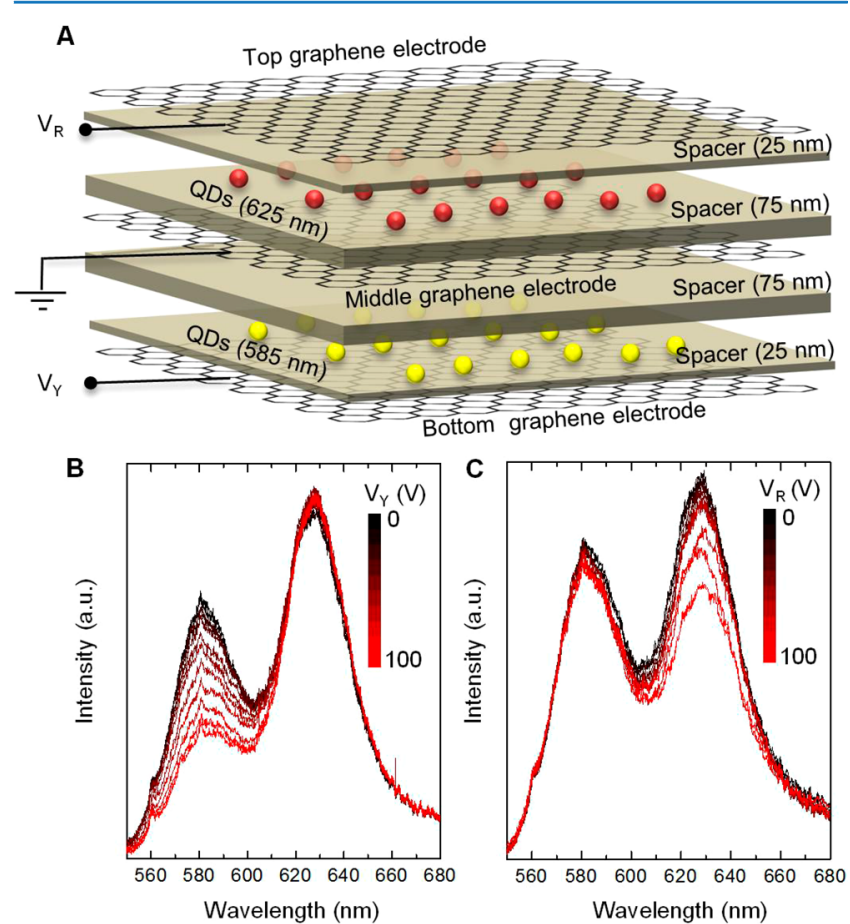

Figure 6. Voltage-controlled color-variable devices. (a) Schematic demonstrates the exploded view of the voltage-controlled colorvariable device that integrates red $(625 \mathrm{~nm})$ and yellow $(585 \mathrm{~nm})$ QDs. (b, c) Recorded spectra of the QDs for various bias conditions. The common middle electrode is grounded. The intensity of yellow (red) QDs is modulated by applying a voltage bias to the bottom (the top) electrodes.

shows the device layout, which combines two graphene capacitors integrated with different QDs having emission wavelengths at 585 and $625 \mathrm{~nm}$. The middle graphene electrode is shared as a common ground electrode. To prevent quenching due to the middle electrode, we deposited thick dielectric layers $(75 \mathrm{~nm})$ on both sides of the electrode. The variation of the carrier density on top and bottom electrodes independently controls the fluorescence intensity of red and yellow QDs. Figure 6 (b and c) shows the fluorescence spectra of the multilayer capacitor for various biasing conditions. We labeled the voltage of the top electrode as $V_{\mathrm{r}}$ and bottom as $V_{\mathrm{y}}$ referring to red and yellow colors, respectively. The red and yellow QDs can be quenched independently by adjusting $V_{\mathrm{r}}$ and bottom as $V_{\mathrm{y}}$. Figure $6 \mathrm{~b}, \mathrm{c}$ shows the recorded spectra as we tuned $V_{\mathrm{y}}\left(V_{\mathrm{r}}\right)$ from 0 to $100 \mathrm{~V}$, while the other electrode was kept at $0 \mathrm{~V}$. This vertically integrated multilayer device demonstrates the working principle of voltage-controlled color variation which could find many applications for display technologies that require multicolor operation from a single pixel.

In summary, we here demonstrate graphene-QDs based fluorescent display and voltage-controlled color-variable hybrid optoelectronic devices working in the visible region. Our study shows that the electrical control of carrier density in graphene together with its optical transparency enables gate-controlled fluorescence of QDs placed in close proximity of the graphene surface. The near-field coupling between QDs and graphene yields a nonradiative energy transfer, which is tuned through controlling charge density on graphene. The presented hybrid optoelectronic devices working in the visible spectrum show the promises of the method which could find immediate applications for light-emission and display technologies. Our method can be scaled down to the single QD level; on the other hand, it can be scaled up to large area applications. In addition, unique mechanical properties of graphene allow us to fabricate the hybrid photonic devices on flexible substrates. Besides applied technologies, we anticipate that the presented technique could provide new practical routes for active control of light-matter interaction at the nanometer scale, which could find new implications in quantum optics, for example, electrically tunable emission of single photon sources. ${ }^{35}$

\section{ASSOCIATED CONTENT}

\section{S Supporting Information}

The Supporting Information is available free of charge on the ACS Publications website at DOI: 10.1021/acsphotonics.8b00163.

Supporting figures (PDF).

Movie 1: Gate-controlled fluorescence of colloidal QDs (ZIP).

Movie 2: Gate-controlled fluorescence of colloidal QDs embedded in a p-type graphene transistors (ZIP).

Movie 3: Graphene-based fluorescence display (ZIP).

Movie 4: Dynamic fluorescence images generated by the display device (ZIP).

Movie 5: Dynamic fluorescence images generated by the display device (ZIP).

\section{AUTHOR INFORMATION}

\section{Corresponding Author}

*E-mail: coskun.kocabas@manchester.ac.uk.

ORCID

Nurbek Kakenov: 0000-0003-2321-6157

Sinan Balci: 0000-0002-9809-8688

Coskun Kocabas: 0000-0003-0831-5552

\section{Notes}

The authors declare no competing financial interest.

\section{ACKNOWLEDGMENTS}

This work is supported by the European Research Council (ERC) Consolidator Grant ERC - 682723 SmartGraphene and Scientific and Technological Research Council of Turkey (TUBITAK) Grant No. 113F278. C.K. acknowledges BAGEP Award of the Science Academy.

\section{REFERENCES}

(1) Kirchain, R.; Kimerling, L. A roadmap for nanophotonics. Nat. Photonics 2007, 1, 303-305. 
(2) Curto, A. G.; Volpe, G.; Taminiau, T. H.; Kreuzer, M. P.; Quidant, R.; van Hulst, N. F. Unidirectional Emission of a Quantum Dot Coupled to a Nanoantenna. Science 2010, 329, 930-933.

(3) Akimov, A. V.; Mukherjee, A.; Yu, C. L.; Chang, D. E.; Zibrov, A. S.; Hemmer, P. R.; Park, H.; Lukin, M. D. Generation of single optical plasmons in metallic nanowires coupled to quantum dots. Nature 2007, 450, 402-406.

(4) Yoshie, T.; Scherer, A.; Hendrickson, J.; Khitrova, G.; Gibbs, H. M.; Rupper, G.; Ell, C.; Shchekin, O. B.; Deppe, D. G. Vacuum Rabi splitting with a single quantum dot in a photonic crystal nanocavity. Nature 2004, 432, 200-203.

(5) Gunter, G.; Anappara, A. A.; Hees, J.; Sell, A.; Biasiol, G.; Sorba, L.; De Liberato, S.; Ciuti, C.; Tredicucci, A.; Leitenstorfer, A.; Huber, R. Sub-cycle switch-on of ultrastrong light-matter interaction. Nature 2009, 458, 178-181.

(6) Pacifici, D.; Lezec, H. J.; Atwater, H. A. All-optical modulation by plasmonic excitation of CdSe quantum dots. Nat. Photonics 2007, 1, 402-406.

(7) Cai, W. S.; Vasudev, A. P.; Brongersma, M. L. Electrically Controlled Nonlinear Generation of Light with Plasmonics. Science 2011, 333, 1720-1723.

(8) Dicken, M. J.; Sweatlock, L. A.; Pacifici, D.; Lezec, H. J.; Bhattacharya, K.; Atwater, H. A. Electrooptic Modulation in Thin Film Barium Titanate Plasmonic Interferometers. Nano Lett. 2008, 8, 4048-4052.

(9) Zhang, X.; Liu, M.; Yin, X. B.; Ulin-Avila, E.; Geng, B. S.; Zentgraf, T.; Ju, L.; Wang, F. A graphene-based broadband optical modulator. Nature 2011, 474, 64-67.

(10) Sensale-Rodriguez, B.; Yan, R. S.; Kelly, M. M.; Fang, T.; Tahy, K.; Hwang, W. S.; Jena, D.; Liu, L.; Xing, H. G. Broadband graphene terahertz modulators enabled by intraband transitions. Nat. Commun. 2012, 3, na.

(11) Sun, Z. P.; Martinez, A.; Wang, F. Optical modulators with 2D layered materials. Nat. Photonics 2016, 10, 227-238.

(12) Majumdar, A.; Kim, J.; Vuckovic, J.; Wang, F. Electrical Control of Silicon Photonic Crystal Cavity by Graphene. Nano Lett. 2013, 13, 515-518.

(13) Gan, X. T.; Shiue, R. J.; Gao, Y. D.; Mak, K. F.; Yao, X. W.; Li, L. Z.; Szep, A.; Walker, D.; Hone, J.; Heinz, T. F.; Englund, D. HighContrast Electrooptic Modulation of a Photonic Crystal Nanocavity by Electrical Gating of Graphene. Nano Lett. 2013, 13, 691-696.

(14) Ju, L.; Geng, B. S.; Horng, J.; Girit, C.; Martin, M.; Hao, Z.; Bechtel, H. A.; Liang, X. G.; Zettl, A.; Shen, Y. R.; Wang, F. Graphene plasmonics for tunable terahertz metamaterials. Nat. Nanotechnol. 2011, 6, 630-634.

(15) Kim, J.; Son, H.; Cho, D. J.; Geng, B. S.; Regan, W.; Shi, S. F.; Kim, K.; Zettl, A.; Shen, Y. R.; Wang, F. Electrical Control of Optical Plasmon Resonance with Graphene. Nano Lett. 2012, 12, 5598-5602.

(16) Yao, Y.; Kats, M. A.; Genevet, P.; Yu, N. F.; Song, Y.; Kong, J.; Capasso, F. Broad Electrical Tuning of Graphene-Loaded Plasmonic Antennas. Nano Lett. 2013, 13, 1257-1264.

(17) Balci, S.; Balci, O.; Kakenov, N.; Atar, F. B.; Kocabas, C. Dynamic tuning of plasmon resonance in the visible using graphene. Opt. Lett. 2016, 41, 1241-1244.

(18) Wang, F.; Zhang, Y. B.; Tian, C. S.; Girit, C.; Zettl, A.; Crommie, M.; Shen, Y. R. Gate-variable optical transitions in graphene. Science 2008, 320, 206-209.

(19) Wang, F.; Chen, C. F.; Park, C. H.; Boudouris, B. W.; Horng, J.; Geng, B. S.; Girit, C.; Zettl, A.; Crommie, M. F.; Segalman, R. A.; Louie, S. G. Controlling inelastic light scattering quantum pathways in graphene. Nature 2011, 471, 617-620.

(20) Li, Z. Q.; Henriksen, E. A.; Jiang, Z.; Hao, Z.; Martin, M. C.; Kim, P.; Stormer, H. L.; Basov, D. N. Dirac charge dynamics in graphene by infrared spectroscopy. Nat. Phys. 2008, 4, 532-535.

(21) Bonaccorso, F.; Sun, Z.; Hasan, T.; Ferrari, A. C. Graphene photonics and optoelectronics. Nat. Photonics 2010, 4, 611-622.

(22) Salihoglu, O.; Kakenov, N.; Balci, O.; Balci, S.; Kocabas, C. Graphene as a Reversible and Spectrally Selective Fluorescence Quencher. Sci. Rep. 2016, 6, 33911.
(23) Lyo, S. K. Energy transfer from an electron-hole plasma layer to a quantum well in semiconductor structures. Phys. Rev. B: Condens. Matter Mater. Phys. 2010, 81, na.

(24) Basko, D.; La Rocca, G. C.; Bassani, F.; Agranovich, V. M. Forster energy transfer from a semiconductor quantum well to an organic material overlayer. Eur. Phys. J. B 1999, 8, 353-362.

(25) Achermann, M.; Petruska, M. A.; Kos, S.; Smith, D. L.; Koleske, D. D.; Klimov, V. I. Energy-transfer pumping of semiconductor nanocrystals using an epitaxial quantum well. Nature 2004, 429, 642646.

(26) Kim, T. H.; Cho, K. S.; Lee, E. K.; Lee, S. J.; Chae, J.; Kim, J. W.; Kim, D. H.; Kwon, J. Y.; Amaratunga, G.; Lee, S. Y.; Choi, B. L.; Kuk, Y.; Kim, J. M.; Kim, K. Full-colour quantum dot displays fabricated by transfer printing. Nat. Photonics 2011, 5, 176-182.

(27) Shirasaki, Y.; Supran, G. J.; Bawendi, M. G.; Bulovic, V. Emergence of colloidal quantum-dot light-emitting technologies. Nat. Photonics 2013, 7, 13-23.

(28) Lee, J.; Bao, W.; Ju, L.; Schuck, P. J.; Wang, F.; Weber-Bargioni, A. Switching Individual Quantum Dot Emission through Electrically Controlling Resonant Energy Transfer to Graphene. Nano Lett. 2014, 14, 7115-7119.

(29) Prasai, D.; Klots, A. R.; Newaz, A. K. M.; Niezgoda, J. S.; Orfield, N. J.; Escobar, C. A.; Wynn, A.; Efimov, A.; Jennings, G. K.; Rosenthal, S. J.; Bolotin, K. I. Electrical Control of near-Field Energy Transfer between Quantum Dots and Two-Dimensional Semiconductors. Nano Lett. 2015, 15, 4374-4380.

(30) Raja, A.; Montoya-Castillo, A.; Zultak, J.; Zhang, X. X.; Ye, Z. L.; Roquelet, C.; Chenet, D. A.; van der Zande, A. M.; Huang, P.; Jockusch, S.; Hone, J.; Reichman, D. R.; Brus, L. E.; Heinz, T. F. Energy Transfer from Quantum Dots to Graphene and MoS2: The Role of Absorption and Screening in Two-Dimensional Materials. Nano Lett. 2016, 16, 2328-2333.

(31) Federspiel, F.; Froehlicher, G.; Nasilowski, M.; Pedetti, S.; Mahmood, A.; Doudin, B.; Park, S.; Lee, J. O.; Halley, D.; Dubertret, B.; Gilliot, P.; Berciaud, S. Distance Dependence of the Energy Transfer Rate from a Single Semiconductor Nanostructure to Graphene. Nano Lett. 2015, 15, 1252-1258.

(32) Tielrooij, K. J.; Orona, L.; Ferrier, A.; Badioli, M.; Navickaite, G.; Coop, S.; Nanot, S.; Kalinic, B.; Cesca, T.; Gaudreau, L.; Ma, Q.; Centeno, A.; Pesquera, A.; Zurutuza, A.; de Riedmatten, H.; Goldner, P.; de Abajo, F. J. G.; Jarillo-Herrero, P.; Koppens, F. H. L. Electrical control of optical emitter relaxation pathways enabled by graphene. Nat. Phys. 2015, 11, 281.

(33) Balci, O.; Kakenov, N.; Karademir, E.; Balci, S.; Cakmakyapan, S.; Polat, E. O.; Caglayan, H.; Özbay, E.; Kocabas, C. Electrically switchable metadevices via graphene. Science Advances 2018, 4, na.

(34) Kasry, A.; Kuroda, M. A.; Martyna, G. J.; Tulevski, G. S.; Bol, A. A. Chemical Doping of Large-Area Stacked Graphene Films for Use as Transparent, Conducting Electrodes. ACS Nano 2010, 4, 3839-3844.

(35) Munnelly, P.; Heindel, T.; Thoma, A.; Kamp, M.; Hofling, S.; Schneider, C.; Reitzenstein, S. Electrically Tunable Single-Photon Source Triggered by a Monolithically Integrated Quantum Dot Microlaser. ACS Photonics 2017, 4, 790-794. 